Daniel Rodrigues Ferreira Saint Martin ${ }^{\mathrm{a}}$ (iD) https://orcid.org/0000-0002-6539-486X Leonardo Correa Segedia,b

(iD) https://orcid.org/0000-0001-5139-2989

Edgard de Melo Keene von Koenig Soares ${ }^{\mathrm{a}}$ (i) https://orcid.org/0000-0002-3696-9556

Rosenkranz Maciel Nogueira,a

(D) https://orcid.org/0000-0002-5254-7601

Carlos Janssen Gomes Cruz ${ }^{\mathrm{a}}$

(iD) https://orcid.org/0000-0002-5879-8102

Keila Elizabeth Fontana ${ }^{a}$

(iD) https://orcid.org/0000-0003-4262-0403

Guilherme Eckhardt Molina ${ }^{\mathrm{a}}$

(iD) https://orcid.org/0000-0002-5937-079X

Luiz Guilherme Grossi Porto ${ }^{\mathrm{a}}$

(iD) https://orcid.org/0000-0002-6240-1614

a Universidade de Brasília (UnB),

Faculdade de Educação Física,

Grupo de Estudos em Fisiologia e

Epidemiologia do Exercício e da

Atividade Física. Brasília, DF, Brasil.

${ }^{\mathrm{b}}$ Corpo de Bombeiros Militar do

Distrito Federal, Grupamento de

Proteção Ambiental. Brasília, DF, Brasil.

Contato:

Luiz Guilherme Grossi Porto

E-mail:

luizggporto@gmail.com

Os autores informam que a pesquisa recebeu apoio do Conselho Nacional de Desenvolvimento Científico e Tecnológico (CNPq), Universal 14/2013 480092/2012-3; e da Fundação de Apoio à Pesquisa do Distrito Federal (FAPDF). O presente trabalho foi realizado também com o apoio da Coordenação de Aperfeiçoamento de Pessoal de Nível Superior (Capes) Código de Financiamento 001 bolsa de mestrado a DRFSM.

Os autores declaram que não há conflitos de interesses.

Os autores informam que este trabalho não foi apresentado em reunião científica.

O trabalho foi baseado na dissertação de mestrado de Daniel Rodrigues Ferreira Saint Martin, intitulada Avaliação objetiva do nível de atividade física, do comportamento sedentário e da aptidão cardiorrespiratória de bombeiros militares, defendida em agosto de 2018 na Universidade de Brasília.

Recebido: 24/09/2018

Revisado: 11/12/2018

Aprovado: 04/02/2019

\section{Nível de atividade física e sobrecarga cardiovascular em bombeiros militares durante combate a incêndio florestal: um estudo exploratório}

\author{
Physical activity level and cardiovascular strain in wildland \\ firefighters during forest fire suppression: an exploratory study
}

\section{Resumo}

Introdução: combates a incêndios florestais expõem bombeiros a elevado risco cardiovascular. Objetivo: avaliar o nível de atividade física (ATF), o comportamento sedentário e a sobrecarga cardiovascular de bombeiros durante um combate a incêndio florestal de grande porte. Métodos: durante 9 dias de atividade, $24 \mathrm{~h} /$ dia, avaliaram-se 7 bombeiros do sexo masculino. Os participantes tiveram a frequência cardíaca $(\mathrm{FC})$ medida a cada segundo e a ATF registrada por um sensor de movimento, do tipo acelerômetro, a cada 60 segundos. Foram computadas as médias dos registros do $2^{\circ}, 3^{\circ}$ e $4^{\circ}$ dias. A ATF e o comportamento sedentário foram avaliados pelo vetor magnitude do acelerômetro, e a sobrecarga cardiovascular, pelo comportamento da FC. Resultados: os participantes apresentaram média de 112 (DP 24) min/dia de ATF moderada/vigorosa (MV), acumularam em média 14.803 (DP 1.667) passos/dia e gasto energético de 1.860 (DP 481) kcal/dia. A sobrecarga cardiovascular permaneceu, em média, 47 (DP 42) min/dia em esforço vigoroso e 8 (DP 12) min/dia em esforço muito vigoroso. Conclusão: os bombeiros avaliados acumularam mais que o dobro da recomendação mínima de ATF-MV semanal em três dias, apresentaram mais de $50 \mathrm{~min} /$ dia de elevada sobrecarga cardiovascular e pouco tempo em comportamento sedentário, se comparados a outras profissões.

Palavras-chave: bombeiros; acelerômetro; incêndio florestal; saúde do trabalhador.

\begin{abstract}
Introduction: wildland fire suppression exposes firefighters to high cardiovascular risk. Objective: to evaluate the physical activity level (PAL), sedentary behavior (SB) and cardiovascular strain of wildland firefighters during a large fire suppression. Methods: for 9 days, 24h/day, we evaluated 7 male wildland firefighters. Participants had their heart rate (HR) measured every second and physical activity recorded by an accelerometer sensor every 60 seconds. The mean records of the $2^{\text {nd }}, 3^{\text {rd }}$ and $4^{\text {th }}$ days of fire suppression were computed for the analysis. PAL was evaluated by the vector magnitude and cardiovascular strain by HR intensity. Results: the volunteers spent on average 112 (SD 24) min/day in moderate to vigorous physical activity (MVPA), achieved 14,803 (SD 1,667) steps/day and spent 1,860 (SD 481) kcal/day. Cardiovascular strain remained on average at 47 (SD 42) $\mathrm{min} /$ day of vigorous effort and 8 (SD 12) min/day of very vigorous physical activity. Conclusion: the firefighters evaluated during a large-scale wildland fire suppression accounted for more than double of the minimum recommended MVPA/week in three days, showed more than $50 \mathrm{~min} /$ day of high cardiovascular strain, and little time on sedentary behavior compared to other professions.
\end{abstract}

Keywords: firefighters; accelerometer; forest fire; occupational health. 


\section{Introdução}

A atividade profissional de bombeiros militares envolve intensas demandas físicas e emocionais, que resultam em elevada sobrecarga cardiovascular; abarca, normalmente, combate a incêndios (urbanos e florestais), resgates e emergências pré-hospitalares $^{1,2}$. Essas atividades expõem bombeiros a diferentes riscos ocupacionais, caracterizando a profissão como de alto risco e com elevada taxa de mortalidade cardiovascular em serviço ${ }^{3-5}$.

Aproximadamente $45 \%$ das mortes de bombeiros americanos em serviço são de origem cardiovascular ${ }^{4}$. Em Massachusetts (Estados Unidos), registraram-se 87 óbitos e 113 afastamentos de bombeiros por causas cardiovasculares em uma década. O número de mortes relacionadas ao combate a incêndio florestal (CIF) e urbano foi proporcionalmente maior que nas outras atividades ocupacionais. Deste número, cerca de 1/3 foram decorrências diretas do incêndio; e $2 / 3$ ocorreram em outras atividades, como na resposta ao brado - o toque da sirene de alerta para atendimento de ocorrências -, no retorno de ocorrências e durante atendimento a emergências médicas. Importante ressaltar que o tempo de permanência dos profissionais no combate a incêndios era significativamente menor que aquele despendido nas outras atividades, demonstrando assim o aumento de risco associado a esta atividade específica ${ }^{6}$.

O CIF requer boa condição física, pois envolve o uso de equipamentos pesados como mochila de água costal, abafadores, enxadas, pás e motosserras ${ }^{7}$. $\mathrm{O}$ peso destas ferramentas oscila entre $3 \mathrm{~kg}$ e $20 \mathrm{~kg}$ e todas são manuseadas em terrenos acidentados e com elevada concentração de gases tóxicos ${ }^{8}$. O CIF pode envolver períodos de 12 a 18 horas de atividade laboral e resultar em gasto energético de 2.400-2.600 kcal $/ \mathrm{dia}^{9}$. Durante o CIF os bombeiros executam um trabalho extenuante, normalmente em regiões de difícil acesso, com temperaturas elevadas, baixíssima umidade relativa do ar e sob condições altamente estressantes ${ }^{10}$. Devido à combinação de fatores ambientais e do esforço físico envolvido, os bombeiros comumente sofrem com o estresse térmico e a desidratação ${ }^{11}$, e evidências demonstram que a atividade pode gerar uma sobrecarga cardiovascular significativa, incluindo alterações na função cardíaca e vascular ${ }^{2,12}$.

O Distrito Federal é uma região com longo período de seca anual e elevado número de incêndios florestais, resultando em intenso recrutamento de bombeiros em ações que podem durar horas, dias ou semanas. Nesse contexto, o Corpo de Bombeiros Militar do Distrito Federal (CBMDF) realiza anualmente (entre maio e novembro), a "Operação Verde Vivo" ${ }^{3}$. Assim, é de extrema importância que se conheça melhor a sobrecarga física e cardiovascular relacionada ao CIF neste cenário, visando possíveis ações de prevenção de agravos associados à atuação profissional ${ }^{14}$.
Em outubro de 2017 houve um grande incêndio florestal no Parque Nacional da Chapada dos Veadeiros, localizado no estado de Goiás. Esta é a maior unidade de conservação de Cerrado no mundo e teve cerca de $30 \%$ de sua área queimada. Mais de 400 brigadistas, bombeiros e voluntários trabalharam no combate às chamas. De acordo com o Instituto Chico Mendes de Conservação da Biodiversidade ${ }^{15}$ esta foi a maior queimada da história do parque e teve, consequentemente, o maior recrutamento de combatentes e aeronaves. O CBMDF colaborou nesta força-tarefa com um grupo de 25 militares.

Sabe-se que, durante CIF, bombeiros enfrentam uma série de dificuldades ocupacionais e ambientais $^{14}$. São necessárias mais informações sobre essas dificuldades, para que haja o desenvolvimento de estratégias de otimização do trabalho e a promoção da saúde de profissionais especialistas em CIF $^{16}$. Praticamente inexistem estudos nacionais que avaliem objetivamente o nível de atividade física (ATF) e o esforço cardiovascular a que bombeiros são expostos durante o CIF. Nesse contexto, o objetivo do estudo foi avaliar o nível de ATF, o número de passos diários, o gasto energético, o comportamento sedentário e o esforço cardiovascular de bombeiros militares em atuação real durante um CIF de grande magnitude.

\section{Métodos}

Realizou-se estudo transversal descritivo, com seleção da amostra por conveniência.

\section{Amostra}

A amostra foi composta por 7 bombeiros do sexo masculino do CBMDF, que se voluntariaram dentre os 25 que compuseram a força tarefa. Tendo em vista a limitação do número de equipamentos disponíveis para o monitoramento das variáveis fisiológicas em campo (7 acelerômetros), definiu-se, como critério de seleção, a ordem de manifestação de concordância em participar.

\section{Dinâmica e organização do trabalho dos participantes}

Os voluntários entraram na força-tarefa dia 20 de outubro, saindo de Brasília com destino ao Parque Nacional da Chapada dos Veadeiros/GO. Segundo recordatório dos voluntários, a rotina diária tinha início às 7h. Após o café da manhã os voluntários se deslocavam de carro ao local de CIF, em trajeto de aproximadamente uma hora. Por volta de $8 \mathrm{~h} 30 \mathrm{~min}$ iniciava-se o trabalho de abertura de caminhos em meio à vegetação e de combate ao incêndio, com reduzida capacidade de ingestão hídrica devido às condições ambientais extremas. Durante o CIF foram utilizados sopradores e mochilas de água costal, o que representava uma carga de equipamentos de até $20 \mathrm{~kg}$. Em todos os dias foram realizados intervalos de $2 \mathrm{~h}$ para almoço/descanso. Outros pequenos períodos de pausa ocorreram 
para orientação e navegação no local, por momentos de exaustão devido ao CIF ou por dificuldades de progressão devido às características da região. $\mathrm{O}$ deslocamento de volta ao alojamento também foi realizado por meio de carros, por volta das $18 \mathrm{~h}$ do mesmo dia.

\section{Instrumentos}

Ainda no quartel, imediatamente antes da partida de Brasília em direção ao foco do incêndio, foram medidas: a frequência cardíaca (FC) em batimentos por minuto (bpm), utilizando um frequencímetro ${ }^{17}$ (H10-Polar $\left.{ }^{\circledR}\right)$; e a pressão arterial de repouso dos voluntários. Um sensor de movimento (acelerômetro-ActiGraph-GT3X $+{ }^{\circledR}$ ) foi utilizado para o registro da ATF. O acelerômetro, posicionado no lado direito da cintura ${ }^{18}$, foi programado para registro dos dados a uma frequência de $60 \mathrm{~Hz}$ e intervalos de gravação de 60 segundos $^{19}$. O acelerômetro registra o tempo e a distância da aceleração/desaceleração contra a gravidade, tendo como referência um ponto fixo no corpo (local de posicionamento do sensor). $\mathrm{O}$ aplicativo do fabricante transforma essas informações em contagens por minuto (cpm - counts per minute). No sensor foram inseridas a massa corporal $(\mathrm{kg})$, a estatura $(\mathrm{cm})$ e a data de nascimento do voluntário. A ATF foi avaliada pelo vetor magnitude do acelerômetro, e a sobrecarga cardiovascular, pelo comportamento da $\mathrm{FC}^{20}$.

Os valores da aptidão cardiorrespiratória (ACR) foram estimados por meio do teste de corrida de Cooper, que é um teste indireto que estima o consumo máximo de oxigênio $\left(\mathrm{VO}_{2} \max \mathrm{em} \mathrm{mL} \cdot \mathrm{kg}^{-1} \bullet \mathrm{min}^{-1}\right)$. O teste é realizado anualmente na corporação durante o teste de aptidão física, tendo sido efetuado cerca de 5 meses antes da atuação no CIF. Para o teste de Cooper, os voluntários foram instruídos a correr o mais rápido possível durante 12 minutos em uma pista de corrida de 400 m. A distância atingida foi convertida em consumo máximo de oxigênio ( $\mathrm{VO}_{2} \mathrm{max}$ ), utilizando a fórmula: $\mathrm{VO}_{2} \max =($ Distância -504$) / 45^{21}$.

A pesquisa foi aprovada pelo Comitê de Ética e Pesquisa em Seres Humanos da Faculdade de Ciências da Saúde da Universidade de Brasília, e todos os participantes assinaram o Termo de Consentimento Livre e Esclarecido (TCLE).

\section{Procedimentos e análise estatística}

Adotou-se como critério a gravação mínima de 10 horas/dia no acelerômetro. Os voluntários utilizaram os aparelhos de medida da FC e da ATF por 9 dias, 24 horas/dia. Contudo, foram analisadas as médias dos registros do $2^{\circ}, 3^{\circ}$ e $4^{\circ}$ dias de CIF por apresentarem registros válidos de todos os voluntários. Excluíram-se da análise o tempo de sono, o período em que o aparelho não registrou nenhuma aceleração (counts) e os registros com intensidade menor que $10 \mathrm{cpm}$. O retorno ao registro e a gravação de dados se dava quando o aparelho reconhecia aceleração/desaceleração com duração mínima de 2 minutos e maior que $10 \mathrm{cpm}^{22}$. O número de passos e o nível de ATF foram avaliados pelo vetor magnitude. Para avaliação da intensidade da ATF por meio da acelerometria, utilizou-se o ponto de corte adaptado de Sasaki e colaboradores $^{23}$ : comportamento sedentário $<200 \mathrm{cpm}$, atividade leve entre 200 e $2.689 \mathrm{cpm}$, moderada ou vigorosa $\geq 2.690 \mathrm{cpm}$.

A caracterização do esforço cardiovascular foi feita por meio do cálculo do percentual da FC máxima predita para a idade. Para as análises foram desconsiderados os registros nulos (valores zero) de FC e valores abaixo de 40 bpm ou acima de 200 bpm, e que se mostrassem como espículas isoladas no registro, indicando provável artefato. A intensidade de esforço (sobrecarga cardiovascular) foi analisada de acordo com o tempo de permanência em 4 zonas de intensidade, a saber: leve ( $\leq 64 \%$ da FCmax), moderada $(64 \%<\mathrm{FC} \leq 76 \%$ da FCmax), vigorosa (76\% < FC < 94\% da FCmax) e muito vigorosa ( $\geq 94 \%$ da $\mathrm{FCmax}^{24}$. Em razão da indisponibilidade de equipamentos, apenas 5 voluntários tiveram a FC monitorada simultaneamente ao uso dos acelerômetros.

O gasto energético foi estimado por meio do software ActiLife v.6 $13.3^{\circledR}$, que se baseia nos dados do eixo vertical do acelerômetro (eixo y) e nos valores de FC, desconsiderando-se o gasto energético basal e aquele referente às atividades leves. A média de calorias gastas em atividade moderada/vigorosa (MV) foi computada utilizando valores de FC maiores ou iguais a $80 \mathrm{bpm}$ e cpm maiores que 1.951.

Para o processamento dos dados, utilizou-se o aplicativo Statistical Package for Social Sciences ${ }^{\mathrm{TM}}$ (SPSS 17.0) para Windows. Após a confirmação dos pressupostos de normalidade, a comparação do tempo despendido em comportamento sedentário, atividade de intensidade leve e MV entre os três dias foi realizada por meio da ANOVA One-Way com post-hoc de Bonferroni $(\mathrm{p}<0,05)$. Os dados são apresentados de forma descritiva, dia a dia e como média dos 3 dias. Os valores são expressos em média e desvio padrão (DP).

\section{Resultados}

Os voluntários apresentaram média de idade de 39,9 (DP 7,3) anos, IMC de 28,1 (DP 3,0) kg/m², FC de repouso de 67 (DP 6,9) bpm, pressão arterial de repouso de 121/78 $\mathrm{mmHg} \mathrm{VO}_{2} \max$ de 43,4 (DP 7,6$) \mathrm{mL} \bullet \mathrm{kg}^{-1} \bullet \mathrm{min}^{-1}$.

A descrição das variáveis "tempo de uso" (tempo de registro diário com dados válidos), comportamento sedentário, atividade leve, MV, número de passos, número de períodos de tempo (bouts) com registro maior que 10 minutos (bouts $>10 \mathrm{~min}$ ) e tempo médio de acúmulo de cada um dos bouts são apresentados na Tabela 1.

Não houve diferença significativa no tempo de comportamento sedentário e de atividade MV entre os diferentes dias. Foi acumulado maior número de passos e maior tempo relativo em atividade de intensidade leve no dia 1 , comparado ao dia $3(p=0,02)$. 
Tabela 1 Caracterização de variáveis-alvo avaliadas por acelerometria em 7 bombeiros militares em operação de combate a incêndio florestal de grande magnitude. Os resultados correspondem à média (DP)

\begin{tabular}{|c|c|c|c|c|c|}
\hline & Dia 1 & $\operatorname{Dia} 2$ & Dia 3 & $p$ & Média $(D P)$ \\
\hline Tempo de uso (min) & $1.104(114)$ & $964(99)$ & $939(151)$ & 0,05 & $995(90)$ \\
\hline Tempo de CS (min) & $300(121)$ & $294(101)$ & $336(95)$ & 0,74 & $305(91)$ \\
\hline Tempo de atividade leve (min) & $696 *(76)$ & $560(57)$ & $484(55)$ & 0,01 & $578(21)$ \\
\hline Tempo de atividade MV (min) & $107(25)$ & $110(34)$ & $119(45)$ & 0,81 & $112(24)$ \\
\hline Número de passos & $\begin{array}{c}16.985^{\#} \\
(1.986)\end{array}$ & $\begin{array}{l}14.060 \\
(2.617)\end{array}$ & $\begin{array}{l}13.448 \\
(2.101)\end{array}$ & 0,02 & $\begin{array}{l}14.803 \\
(1.667)\end{array}$ \\
\hline Número de bouts de atividade MV ( $\geq 10 \mathrm{~min})$ & $2(1)$ & $2(2)$ & $1(1)$ & 0,49 & $2(1)$ \\
\hline Tempo de bouts de atividade MV ( $\geq 10 \mathrm{~min}$ ) & $30(24)$ & $28(24)$ & $18(13)$ & 0,52 & $25(10)$ \\
\hline CS $(\%)$ & $27(8)$ & $30(9)$ & $35(6)$ & 0,15 & $31(8)$ \\
\hline Atividade leve (\%) & $63^{\#}(7)$ & $59(8)$ & $52(5)$ & 0,02 & $58(8)$ \\
\hline Atividade MV (\%) & $10(3)$ & $11(4)$ & $13(4)$ & 0,44 & $11(4)$ \\
\hline
\end{tabular}

MV: moderada/vigorosa; min: minutos; CS: comportamento sedentário; ": dia 1 diferente dos demais; \#: dia 1 diferente do dia 3.

Teste paramétrico ANOVA.

\section{Frequência cardíaca}

O tempo médio de registro da FC foi de 17 horas e 18 minutos, sendo 15 horas e 12 minutos em atividade de intensidade leve e 2 horas e 6 minutos em MV. O tempo de permanência na intensidade vigorosa oscilou entre 3 e 113 minutos. O tempo médio de permanência na zona de intensidade muito vigorosa oscilou entre 0 e 33 minutos, ressaltando-se que este tempo é a soma de todos os momentos que a FC do voluntário atingiu tal intensidade. A descrição do tempo de permanência em cada zona de intensidade, de acordo com o comportamento da FC, é apresentada na Tabela 2. Foi observada uma grande dispersão dos valores apresentados para algumas variáveis, provavelmente devido à amostra limitada.

Tabela 2 Valores absolutos e relativos da intensidade da atividade física avaliada pela frequência cardíaca e da estimativa do gasto calórico em 5 bombeiros militares em operação de combate a incêndio florestal de grande magnitude. Os resultados correspondem à média (DP)

\begin{tabular}{lccccc}
\hline & Dia 1 & Dia 2 & Dia 3 & $p$ & Média (DP) \\
\hline Tempo de uso (min) & $1.055(91)$ & $1.073(111)$ & $886(324)$ & 0,2 & $1.038(119)$ \\
Tempo de atividade leve (min) & $948(124)$ & $883(124)$ & $760(290)$ & 0,25 & $912(125)$ \\
Tempo de atividade moderada (min) & $61(50)$ & $96(49)$ & $67(50)$ & 0,1 & $71(45)$ \\
Tempo de atividade vigorosa (min) & $40(60)$ & $80(54)$ & $53(71)$ & 0,39 & $47(42)$ \\
Tempo de atividade muito vigorosa (min) & $6(8)$ & $14(27)$ & $6(10)$ & 0,37 & $8(12)$ \\
Tempo de atividade MV (min) & $107(110)$ & $190(69)$ & $126(89)$ & 0,16 & $126(77)$ \\
Gasto energético em atividade MV (kcal) & $1.860(538)$ & $1.685(452)$ & $1.554(367)$ & 0,52 & $1.860(481)$ \\
Atividade leve (\%) & $90(10)$ & $82(7)$ & $87(10)$ & 0,2 & $88(7)$ \\
Atividade moderada (\%) & $6(5)$ & $9(4)$ & $7(6)$ & 0,15 & $7(4)$ \\
Atividade vigorosa (\%) & $4(5)$ & $7(5)$ & $6(7)$ & 0,41 & $6(4)$ \\
Atividade muito vigorosa (\%) & $1(1)$ & $1(2)$ & $13(1)$ & 0,35 & $1(1)$ \\
Atividade MV (\%) & $10(10)$ & $18(7)$ & 0,2 & $12(7)$ \\
\hline
\end{tabular}

Leve: $\leq 63 \%$ da FCmax; moderado: > 63\% e $\leq 76 \%$ da FCmax; vigoroso: > 76\% e < 94\% da FCmax; muito vigoroso: $\geq 94 \%$ da FCmax; MV: moderada/vigorosa. Teste paramétrico ANOVA. 


\section{Discussão}

Neste estudo com bombeiros militares durante CIF de grandes proporções, observou-se grande acúmulo de ATF durante o serviço, avaliada objetivamente por acelerometria. Quando a intensidade de esforço foi avaliada por zona de FC, observou-se permanência média de 47 minutos/dia em atividade de intensidade vigorosa e 8 minutos/dia em atividades muito vigorosas. Com base na acelerometria, os voluntários acumularam 337 (DP 71) minutos em atividade MV durante os 3 dias, o que corresponde a aproximadamente 2,2 vezes mais atividade MV que o mínimo semanal de 150 minutos de atividade física MV recomendado pela Organização Mundial da Saúde ${ }^{25}$.

Poucos estudos têm avaliado a ATF e o comportamento sedentário no ambiente ocupacional. Destaca-se uma pesquisa que avaliou essas variáveis em 40 profissões ${ }^{26}$, na qual se evidenciou que as profissões de agricultor, enfermeiro e garçom apresentam menor tempo em comportamento sedentário (cerca de 40\%). Cozinheiros, garçons e operadores de máquina permanecem em média $37 \%$ em atividade de intensidade leve; e agricultores, pescadores e funcionários da construção civil permanecem em média 7\% em atividade MV. Em nosso estudo, foram contabilizados $30 \%$ do dia dos bombeiros em comportamento sedentário, 59\% em atividade leve e $11 \%$ em MV. Estes dados demonstram que os bombeiros avaliados tiveram menos tempo de comportamento sedentário e mais atividade leve que as 40 profissões avaliadas em outro estudo ${ }^{26}$, além de um percentual de atividades MV 36\% maior que as profissões que apresentaram maior tempo de atividades MV. Apesar dos registros serem relativos ao desempenho profissional em situação específica, em regime de força-tarefa, as ações desempenhadas pelos voluntários foram semelhantes a outras atividades de rotina realizadas durante a Operação Verde Vivo. Ressalvadas as limitações do tamanho da amostra e a especificidade do regime de força tarefa, os achados demonstram que a atividade de bombeiro no CIF envolve a execução de elevada quantidade de ATF e em elevadas intensidades, se comparada a outras profissões.

Estudo nacional caracterizou o nível de ATF e o comportamento sedentário semanal de habitantes do sul do país e demonstrou uma média de 7 horas e 53 minutos/dia em comportamento sedentário, 5 horas e 25 minutos/dia em atividade de intensidade leve e 30 minutos/dia em atividade $\mathrm{MV}^{27}$. Destaca-se, novamente, que os bombeiros acumularam menos tempo em comportamento sedentário e mais em atividades leves e MV, se comparados à população geral.
A análise da ATF com base no total de passos diários acumulados reforça o entendimento de que o bombeiro envolvido em CIF desenvolve uma ATF muito demandante. Além de uma média de 14.803 passos/dia, destaca-se que 6 dos 7 voluntários monitorados alcançaram média superior ao mínimo recomendado para a saúde, de 10 mil passos/dia ${ }^{28}$, e o sétimo alcançou valor muito próximo (9.906 passos/dia). Destaca-se que esses passos foram acumulados durante o trabalho. Quando se avaliou a cadência de passos por hora, considerando-se o período entre às $8 \mathrm{~h}$ e às $18 \mathrm{~h}$, observou-se que os bombeiros acumularam média de 902 (DP 145) passos/hora durante o expediente, o que é mais que o dobro dos valores encontrados entre trabalhadores administrativos (444 passos/hora) ${ }^{29}$. E até mesmo quando comparados a outros trabalhadores da segurança pública: os bombeiros avaliados apresentaram cadência de passos por hora $45 \%$ maior que de policiais (902 vs 497, respectivamente) ${ }^{30}$.

Além do alto grau de movimentação durante a ação laboral, é importante destacar que sua associação com a intensidade do esforço, o calor extremo e a baixa humidade do ar expõe os bombeiros a riscos elevados. Durante avaliação dos acidentes não fatais ocorridos no decorrer de 867 CIF nos Estados Unidos, foi demonstrado que os incêndios de médio e grande porte resultaram em maior número de lesões entre os combatentes, provavelmente devido ao longo tempo de combate e ao comportamento agressivo do fogo ${ }^{31}$. Não houve relato de lesões entre os voluntários aqui avaliados. É possível que a boa ACR e o bom preparo físico dos bombeiros tenham sido essenciais para o cumprimento da tarefa em segurança e sem lesões. A caracterização do esforço avaliada pela FC demonstrou que, nos 3 dias avaliados, a intensidade vigorosa foi atingida em 93\% dos casos. Já a intensidade muito vigorosa foi alcançada em $47 \%$ das ocasiões. O estudo de Parker e colaboradores $^{32}$ monitorou dois indivíduos em um CIF. Os autores observaram que durante 1 hora e 50 minutos de trabalho em terreno irregular, a FCmax registrada foi $185 \mathrm{bpm}$; e em 50 minutos de trabalho em terreno plano, a FCmax registrada foi $144 \mathrm{bpm}$. O conjunto dos achados demonstra a elevada demanda física associada ao CIF.

No presente estudo, os bombeiros apresentaram um gasto energético médio em atividade de $1.860 \mathrm{kcal} / \mathrm{dia}$, que foi inferior ao observado por Ruby e colaboradores ${ }^{9}$ (2.628 kcal/dia) em combatentes durante atividade de CIF. Essa diferença pode ser justificada pela metodologia adotada para a estimativa do gasto energético, visto que no estudo mencionado utilizou-se a técnica da água duplamente marcada. Deve-se considerar ainda a não contabilização, no presente estudo, do gasto energético em 
atividades de intensidade leve e o uso do acelerômetro na cintura, o que impossibilita o reconhecimento de movimentos realizados pelos membros superiores $^{7}$, que é bastante frequente no CIF, como ao manusear abafadores.

Entre as principais limitações deste estudo, deve-se considerar o tamanho da amostra e a amostragem por conveniência. Entretanto, priorizou-se a monitoração contínua e de forma objetiva em condição muito específica, como um CIF de grandes proporções. A consistência dos resultados quanto às diferentes análises (nível de $\mathrm{ATF}$ /intensidade por minutos/dia; por passos/dia e/ou pela FC) sugere que o reduzido número amostral representa uma limitação maior em análises de associação e de subcategorias do que na própria caracterização dos fenômenos avaliados. O tamanho da amostra também suscita dúvidas quanto ao melhor modelo estatístico a ser empregado, se paramétrico ou não-paramétrico. Optou-se por aplicar testes paramétricos nas análises das variáveis que passaram no teste de normalidade, até porque foram maioria. As poucas variáveis que apresentaram distribuição não-paramétrica não mostraram diferenças estatísticas em qualquer uma das duas abordagens. Vale destacar que as atividades monitoradas ocorreram em regime de força-tarefa, o que pode diferir das ações de rotina ao longo do ano. A avaliação do grau de esforço/sobrecarga ao longo do ano em trabalho rotineiro ultrapassa os objetivos deste estudo e requer futuras investigações. Ademais, não se avaliou aspectos como a intensidade do fogo, a temperatura ambiente ou o grau de hidratação, o que ajudaria sobremaneira na interpretação da sobrecarga laboral em situações de CIF.

Por fim, os bombeiros militares avaliados neste estudo em atividade laboral de CIF de grande porte apresentaram pouco comportamento sedentário, grande quantidade de ATF, elevado gasto energético e estavam sujeitos a elevadas intensidades de esforço físico. Os possíveis impactos de longo prazo na saúde desses trabalhadores avaliados devem ser alvo de investigação, uma vez que a literatura já evidencia fatores de risco cardiovascular crônicos associados à atividade de bombeiro ${ }^{1}$. Os achados sugerem que é preciso uma avaliação contínua de estratégias de intervenção com foco na redução dos riscos à saúde desses profissionais e a necessidade de treinamento físico contínuo de bombeiros com vistas a um nível de aptidão física que diminua a sobrecarga relativa imposta pelo trabalho, promovendo, assim, a saúde e o desempenho profissional daqueles que têm como missão última salvar vidas.

\section{Agradecimentos}

Os autores agradecem aos voluntários da pesquisa e o apoio do Corpo de Bombeiros Militar do Distrito Federal para a realização da pesquisa.

\section{Contribuições de autoria}

Saint-Martin DRF contribuiu com a concepção do estudo, liderou a coleta e análise dos dados, redigiu a versão inicial do manuscrito e revisou a versão final. Segedi LC contribuiu com a concepção do estudo, apoiou na coleta e análise dos dados e na revisão do manuscrito. Soares EMKVK contribuiu com a concepção do estudo, análise dos dados e revisão do manuscrito. Nogueira RM apoiou na coleta de dados, contribuiu com a concepção do estudo, análise dos dados e revisão do manuscrito. Cruz CJG contribuiu com a concepção do estudo, análise dos dados e revisão do manuscrito. Fontana KE contribuiu com a concepção do estudo e revisão do manuscrito. Molina GE contribuiu com a concepção do estudo, análise dos dados e revisão do manuscrito. Porto LGG apoiou na coleta de dados, contribuiu com a concepção e orientação do estudo, análise dos dados, redação e revisão final do manuscrito. Todos os autores assumem responsabilidade pública pelo conteúdo do trabalho.

\section{Referências}

1. Soteriades ES, Smith DL, Tsismenakis AJ, Baur DM, Kales SN. Cardiovascular disease in US firefighters: a systematic review. Cardiol Rev. 2011;19(4):202-15.

2. Smith DL, DeBlois JP, Kales SN, Horn GP. Cardiovascular strain of firefighting and the risk of sudden cardiac events. Exerc Sport Sci Rev. 2016;44(3):90-7.

3. Al-Zaiti SS, Carey MG. The prevalence of clinical and electrocardiographic risk factors of cardiovascular death among on-duty professional firefighters. J Cardiovasc Nurs. 2015;30(5):440-6.

4. Kales SN, Soteriades ES, Christophi CA, Christiani DC. Emergency duties and deaths from heart disease among firefighters in the United States. N Engl J Med. 2007;356(12):1207-15.

5. Yang J, Teehan D, Farioli A, Baur DM, Smith D, Kales SN. Sudden cardiac death among firefighters $\leq 45$ years of age in the United States. Am J Cardiol. 2013;112(12):1962-7. 
6. Geibe JR, Holder J, Peeples L, Kinney AM, Burress JW, Kales SN. Predictors of on-duty coronary events in male firefighters in the United States. Am J Cardiol. 2008;101(5):585-9.

7. Heil DP. Estimating energy expenditure in wildland fire fighters using a physical activity monitor. Appl Ergon. 2002;33(5):405-13.

8. Wegesser TC, Pinkerton KE, Last JA. California Wildfires of 2008: coarse and fine particulate matter toxicity. Environ Health Perspect. 2009;117(6):893-7.

9. Ruby BC, Shriver TC, Zderic TW, Sharkey BJ, Burks C, Tysk S. Total energy expenditure during arduous wildfire suppression. Med Sci Sports Exerc. 2002;34(6):1048-54.

10. Smith DL, Petruzzello SJ, Goldstein E, Ahmad U, Tangella K, Freund GG, et al. Effect of live-fire training drills on firefighters' platelet number and function. Prehosp Emerg Care. 2011;15(2):233-9.

11. Eastlake AC, Knipper BS, He X, Alexander BM, Davis KG. Lifestyle and safety practices of firefighters and their relation to cardiovascular risk factors. Work. 2015;50(2):285-94.

12. Porto LGG, Schmidt ACB, Souza JM, Nogueira RM, Fontana KE, Molina GE, et al. Firefighters' basal cardiac autonomic function and its associations with cardiorespiratory fitness. Work. 2019;62(3):485-95.

13. Corpo de Bombeiros Militar do Distrito Federal. Bolet Comando Inform [Internet]. 2017 [citado em 27 maio 2020];135:3. Disponível em: https://www. cbm.df.gov.br/2012-11-12-17-42-33/2012-11-13-1614-57?task= document.viewdoc\&id $=11752$

14. Aisbett B, Wolkow A, Sprajcer M, Ferguson SA. "Awake, smoky, and hot": providing an evidencebase for managing the risks associated with occupational stressors encountered by wildland firefighters. Appl Ergon. 2012;43(5):916-25.

15. Instituto Chico Mendes de Conservação da Biodiversidade. Chapada dos Veadeiros comemora aniversário [Internet]. 11 jan 2018 [citado em 27 maio 2020]. Disponível em: https://www.icmbio. gov.br/portal/ultimas-noticias/20-geral/9408parque-da-chapada-comemora-seu-aniversario

16. Rodríguez-Marroyo JA, López-Satue J, Pernía R, Carballo B, García-López J, Foster C, et al. Physiological work demands of Spanish wildland firefighters during wildfire suppression. Int Arch Occup Environ Health. 2012;85(2):221-8.

17. Porto LGG, Junqueira Jr LF. Comparison of timedomain short-term heart interval variability analysis using a wrist-worn heart rate monitor and the conventional electrocardiogram. Pacing Clin Electrophysiol. 2009;32(1):43-51.

18. Freedson PS, Melanson E, Sirard J. Calibration of the Computer Science and Applications, Inc. accelerometer. Med Sci Sports Exerc. 1998;30(5):777-81.

19. Lugade V, Fortune E, Morrow M, Kaufman K. Validity of using tri-axial accelerometers to measure human movement-Part I: posture and movement detection. Med Eng Phys. 2014;36(2):169-76.

20. White T, Westgate K, Wareham NJ, Brage S. Estimation of physical activity energy expenditure during free-living from wrist accelerometry in UK adults. PLoS One. 2016;11(12):e0167472.

21. American College of Sports Medicine. ACSM's health-related physical fitness assessment manual. Philadelphia: Lippincott Williams \& Wilkins; 2013.

22. Troiano RP, Berrigan D, Dodd KW, Mâsse LC, Tilert T, Mcdowell M. Physical activity in the United States measured by accelerometer. Med Sci Sports Exerc. 2008;40(1):181-8.

23. Sasaki JE, John D, Freedson PS. Validation and comparison of ActiGraph activity monitors. J Sci Med Sport. 2011;14(5):411-6.

24. American College of Sports Medicine. ACSM's Guidelines for Exercise Testing and Prescription. 7a ed. Philadelphia: Lippincott Williams \& Wilkins; 2006.

25. World Health Organization. Global recommendations on physical activity for health [Internet]. Geneva; 2010 [citado em 27 maio 2020]. Disponível em: https://apps.who.int/iris/bitstream/ handle/10665/44399/9789241599979_eng. pdf?sequence $=1$

26. Steeves JA, Tudor-Locke C, Murphy RA, King GA, Fitzhugh EC, Harris TB. Classification of occupational activity categories using accelerometry: NHANES 2003-2004. Int J Behav Nutr Phys Act. 2015 Jun 30;12:89.

27. Gonçalves PB, Hallal PC, Hino AAF, Reis RS. Individual and environmental correlates of objectively measured physical activity and sedentary time in adults from Curitiba, Brazil. Int J Public Health. 2017;62(7):831-40.

28. Tudor-Locke C, Craig CL, Aoyagi Y, Bell RC, Croteau KA, De Bourdeaudhuij I, et al. How many steps/day are enough? For older adults and special populations. Int J Behav Nutr Phys Act. 2011 Jul 28;8:80.

29. Fisher A, Ucci M, Smith L, Sawyer A, Spinney R, Konstantatou M, et al. Associations between the objectively measured office environment and workplace step count and sitting time: crosssectional analyses from the active buildings study. Int J Environ Res Public Health. 2018;15(6):1135.

30. Ramey SL, Perkhounkova RNY, Moon M, Tseng RNHC, Wilson RNA, Hein MRN, et al. Physical activity in police beyond self-report. J Occup Environ Med. 2014;56(3):338-43.

31. Britton C, Lynch CF, Torner J, Peek-Asa C. Fire characteristics associated with firefighter injury on large federal wildland fires. Ann Epidemiol. 2013;23(2):37-42.

32. Parker R, Vitalis A, Walker R, Riley D, Pearce HG. Measuring wildland fire fighter performance with wearable technology. Appl Ergon. 2017;59 